$\begin{array}{cl}\begin{array}{c}\text { Revue } \\ \text { de I'histoire } \\ \text { des religions }\end{array} & \text { Revue de l'histoire des religions } \\ & \begin{array}{l}\text { Civitas confusionis en débat } \\ \end{array}\end{array}$

\title{
Marie-Hélène BLANCHET et IonuŢ-Alexandru TUDORIE (dir.), L'apport des Assomptionnistes français aux études byzantines : une approche critique
}

Actes du colloque de Bucarest, 25-27 septembre 2014, Paris, Institut français d'études byzantines (« Archives de l'Orient Chrétien », 21), 2017

\section{Antonio Rigo}

\section{OpenEdition Journals}

\section{Édition électronique}

URL : http://journals.openedition.org/rhr/11144

DOl : $10.4000 /$ rhr. 11144

ISSN : 2105-2573

\section{Éditeur}

Armand Colin

\section{Édition imprimée}

Date de publication : 1 mars 2021

Pagination : 173-176

ISBN : 978-2-200-93375-3

ISSN : 0035-1423

\section{Référence électronique}

Antonio Rigo, « Marie-Hélène Blanchet et lonuț-Alexandru Tudorie (dir.), L'apport des Assomptionnistes français aux études byzantines : une approche critique », Revue de l'histoire des religions [En ligne], 1 | 2021, mis en ligne le 19 mars 2021, consulté le 31 mars 2021. URL : http://journals.openedition.org/ rhr/11144; DOl : https://doi.org/10.4000/rhr.11144

Ce document a été généré automatiquement le 31 mars 2021.

Tous droits réservés 
Marie-Hélène BLANCHET et IonuŢAlexandru TUDoRIE (dir.), L'apport des Assomptionnistes français aux études byzantines : une approche critique

Actes du colloque de Bucarest, 25-27 septembre 2014, Paris, Institut

français d'études byzantines (« Archives de l'Orient Chrétien », 21), 2017

\section{Antonio Rigo}

\section{RÉFÉRENCE}

Marie-Hélène BLANCHET et IonuŢ-Alexandru TUDORIE (dir.), L'apport des Assomptionnistes français aux études byzantines : une approche critique. Actes du colloque de Bucarest, 25-27 septembre 2014, Paris, Institut français d'études byzantines (« Archives de l'Orient Chrétien », 21), 2017, 536 p., 24 cm, 80 €, ISBN 978-90-429-3524-2.

1 Tout commence le 7 octobre 1895, lorsque la Congrégation des Assomptionnistes ouvre un Centre d'études orientales à Kadiköy, sur la rive asiatique de Constantinople, qui devient en réalité un Institut d'études byzantines (ensuite IFEB). Exactement cent ans plus tard, en 1995, l'Institut s'installe à l'Institut catholique de Paris, clôturant apparemment une aventure intellectuelle, scientifique et spirituelle qui l'avait mené de l'ancienne Chalcédoine à Bucarest et enfin à Paris. À cette occasion, Albert Failler, « le dernier maillon de la chaîne ", selon ses propres mots, publiait un article important, pénétrant et profond sur ces cent ans, mais non sans amertume quand il observait que le transfert de la bibliothèque à l'Institut Catholique de Paris marquait la fin de l'œuvre et la dissolution de l'Institut et il se désignait comme son " exécuteur testamentaire » ( Le centenaire de l'Institut byzantin des Assomptionnistes », Revue des études byzantines, 53, 1995, p. 5-40). 
2 L'article court mais dense d'A. Failler, qui consistait en « un bref historique de l'Institut, à travers ses sièges successifs » et dans une illustration de ses publications et de la bibliothèque, est maintenant suivi d'un volume qui recueille les interventions d'une quinzaine de spécialistes (M.-H. Blanchet, I.-A.Tudorie, Giuseppe-Maria Croce, Matthieu Cassin, A. Failler, Daniel Galadza, Ivana Jevtić, Vassa Kontouma, Marie-Hélène Congourdeau, Ekaterini Mitsiou, Peter Schreiner, Vangélis Maladakis, Vivien Prigent, Cécile Morrisson, Christian Gastgeber) à l'occasion d'un colloque consacré précisément à l'histoire et à l'apport scientifique de l'Institut et de ses membres au cours du $\mathrm{xx}^{\mathrm{e}}$ siècle. La pluralité des voix ne compromet pas l'unité et la cohérence du volume ni la facilité de lecture, malgré l'ampleur de certaines contributions (les pages de M. Cassin sur la bibliothèque, celles de V. Prigent sur la sigillographie) et la quantité considérable de nouveaux matériaux présentés ici. Il s'agit vraiment d'un beau livre, fascinant pour l'histoire intellectuelle et religieuse de l'époque contemporaine. Il est divisé en deux grandes parties, dont la première (historique) concerne l'histoire de l'Institut, ses vicissitudes de Kadiköy (1895-1937) à Bucarest (1937-1947) et enfin à Paris (1947-1995), ses membres, ses lieux, sa bibliothèque et ses livres, tandis que la seconde (historiographique) traite de différents domaines et sujets d'étude (épigraphie, diplomatique, sigillographie, etc.) sur lesquels se sont concentrés les membres de l'Institut.

3 Le titre général du livre ne reflète en réalité qu'un seul - certes le principal - des deux parcours tracés dans le volume. En effet, il suffit d'évoquer ici ne fût-ce que les noms de Louis Petit, Vitalien Laurent et Jean Darrouzès, pour se rendre immédiatement compte de la contribution décisive et prolongée de l'Institut aux études sur Byzance. Mais en parallèle, ou plutôt au prisme des événements et des hommes liés à l'Institut, le volume retrace également l'itinéraire de la Constantinople ottomane puis kemaliste, jusqu'à Paris en passant par la Bucarest de Charles II (avec la guerre et l'arrivée de l'Armée Rouge). Il déroule aussi une partie importante de l'histoire de l'Église à l'époque moderne et contemporaine, en particulier l'attitude de Rome envers le christianisme oriental, de l'encyclique de Pie IX Ad Orientales (1848) au concile Vatican II et au-delà (comme le rappelle G.-M. Croce).

4 La naissance de l'Institut s'inscrivait plus généralement dans une politique de retour des Orientaux à Rome et elle était spécifiquement liée à la mission du pape Léon XIII concernant l'étude du christianisme oriental, toujours avec l'idée d'un ralliement des dissidents à l'Église romaine. Mais, « débarqués d'un monde étranger et n'ayant pour armes que leur zèle ultramontain et leur science, les fondateurs se rendirent rapidement compte que la connaissance doit précéder l'action et, une fois la connaissance acquise, ils semblent avoir douté que l'action qu'on leur proposait de mener fût possible ou même souhaitable... C'est ainsi que les apôtres se firent érudits. L'histoire de l'Institut, jusqu'à ses derniers avatars, reflète ce dilemme " (Failler, "Le centenaire», p. 6). En effet, les cent ans relatés dans le volume témoignent de cette tension, liée selon toute probabilité à l'ambiguïté ou, mieux, à la duplicité du projet initial (voir les remarques des éditeurs dans l'Introduction, p. 22), qui est évidemment à l'origine des nombreuses interventions répétées au fil des ans par la Congrégation. Les supérieurs ont par la suite engagé l'Institut dans une voie sans issue, en décrétant effectivement sa fin. En premier lieu, «La question de l'œcuménisme a été en effet au centre de la contestation portée par les supérieurs religieux sur les travaux et le fonctionnement de l'IFEB. Les autorités de la congrégation ne voyaient pas l'intérêt de 
recherches qui n'étaient pas directement exploitées dans le dialogue œcuménique du jour » (p. 229). Mais de plus, selon une attitude et un jugement récurrent dans certains milieux religieux catholiques, on affirmait que les travaux de Vitalien Laurent sur la sigillographie, ceux de Venance Grumel sur la chronologie, ceux de Raymond Janin sur la topographie de Constantinople, étaient une "occupation vaine et profane » et d'inutiles « amusements de collectionneur » (mémoire de V. Grumel, 1960).

5 Les recherches scientifiques de l'Institut pendant un siècle se sont inscrites par la volonté des initiateurs (Louis Petit d'abord, Vitalien Laurent ensuite) dans un projet ambitieux plus général visant à la refonte de l'Oriens Christianus de Michel Le Quien, et aussi de la Constantinopolis christiana de Charles Du Cange. Ce projet est réaffirmé à plusieurs reprises dans divers articles parus dans la revue de l'Institut publiée d'abord à Kadiköy puis à Bucarest, les Échos d'Orient, et également plus tard dans la Revue des études byzantines. Cette idée, qui était en quelque sorte liée aux travaux des érudits catholiques du xviI ${ }^{\mathrm{e}}$ siècle (voir l'Introduction, p. 19), n'a jamais atteint sa pleine et entière réalisation, mais nombre de contributions monumentales des membres de l'Institut, qui sont aujourd'hui des ouvrages de référence pour les études byzantines, faisaient évidemment partie de ce plan général (les Régestes du patriarcat de Constantinople, les Notitiae episcopatuum, les volumes sur la géographie ecclésiastique byzantine, sur la topographie de Constantinople, etc.).

6 Le cœur des études de l'Institut, attesté non seulement par les œuvres majeures mais aussi par les deux revues successives qui ont longtemps été l'organe de l'équipe, a toujours été l'histoire de l'Église byzantine conçue surtout comme institution, et avec certes moins d'attention (à quelques exceptions près) aux doctrines et à la théologie, et donc aux «thèmes brûlants » de la polémique entre les Byzantins et les Occidentaux, étudiée plutôt dans sa dimension historique et ecclésiale. L'itinéraire de Martin Jugie (voir la contribution de V. Kontouma), ainsi que quelques événements mineurs, sont à cet égard emblématiques. Les travaux de M. Jugie (pensons aux volumes sur la théologie dogmatique des Orientaux et aux articles dans le Dictionnaire de théologie catholique) et ses interventions sur ces sujets sont en fait postérieurs à son départ de l'Institut et coïncident avec sa période romaine à l'Institut Pontifical Oriental.

7 Si la théologie au sens strict du terme n'a jamais fait partie des sujets privilégiés dans les recherches de l'équipe assomptionniste, il fautage au contraire souligner l'attention prolongée, à partir des années $30 \mathrm{du} \mathrm{xx}{ }^{\mathrm{e}}$ siècle, à la spiritualité byzantine et postbyzantine. À cet égard, on ne peut s'empêcher de rappeler la figure de Jean Gouillard (même si le livre ne se focalise pas sur lui) et ses articles dans les Échos d'Orient de ces années-là et, après sa sortie de l'Institut, sa Petite Philocalie de la prière du cœur (1953), livret qui devait avoir un retentissement significatif au niveau international. Nous devons également au même savant l'édition du Synodikon de l'orthodoxie (1967), document central de l'histoire et des doctrines de l'Église byzantine, et ancien projet de l'équipe assomptionniste lié à la refonte de l'Oriens Christianus.

8 Les cas de Martin Jugie et Jean Gouillard ont montré, sous des formes et par des voies certainement différentes, le rayonnement direct et personnel du travail de l'Institut dont nous avons des témoignages, certainement plus distants, jusque dans de nombreux projets de recherche récents ou en cours, sur les règlements monastiques, les typika (E. Mitsiou), sur le registre patriarcal de Constantinople (Ch. Gastgeber), sur la sigillographie (V.Prigent), sur les monastères du Mont Athos et leurs archives (V. Maladakis - cf. aussi le récent article d'Olivier Delouis, « Un archevêque latin sur le 
Mont Athos (mai-juillet 1921) : Mgr Louis Petit d'après son secrétaire Jules Pector ", Travaux et Mémoires, 23/2, 2019, p.507-526). Cette puissante influence s'est aussi matérialisée de manière plus profonde et peut-être à première vue de manière moins évidente dans la structuration de la recherche. Le fait est que de nombreux membres de l'équipe assomptionniste de l'Institut ont concentré leurs travaux sur l'ère des Paléologues pendant de nombreuses années. À l'heure actuelle, les byzantinistes français disposent d'un centre de recherche au niveau international consacré précisément à cette époque. Évidemment, ce n'est pas un hasard, mais plutôt une preuve de l'héritage durable de l'équipe des Assomptionnistes.

9 M.-H. Blanchet et I.-A. Tudorie commencent l'introduction du volume par ces mots : «L'historiographie devrait toujours se garder d'être soit un exercice d'admiration, soit l'occasion d'une trop facile critique ». L'ouvrage, sous-titré " Une approche critique », parvient à maintenir pleinement et heureusement cet équilibre, sans tomber dans l'éloge et l'hagiographie. L'auteur de cette critique espère pour sa part avoir réussi dans son entreprise.

\section{AUTEURS}

\section{ANTONIO RIGO}

Università Ca' Foscari, Venise. 